\title{
LA CATTEDRALE, CUORE RELIGIOSO DELLA CITTÀ
}

\author{
PAOLA VISMARA $(*)$
}

RiassunTo. - Si ripercorrono alcune tappe del ruolo del Duomo di Milano nella storia della città, per grandissime linee. In tale sede, almeno sino alla fine dell'ancien régime, avevano luogo i grandi eventi della vita politica e civile, seppur non senza tensioni. La cattedrale era il cuore della città, in primo luogo il cuore liturgico e pastorale della vita religiosa. Si segnala lo sfarzo delle cerimonie straordinarie che vi si svolgevano, il ruolo della musica e, in particolare, la funzione del luogo e delle sue cerimonie nel contesto dell'azione degli arcivescovi. Seppur in forme diverse rispetto al passato, alcuni aspetti della ritualità e della centralità del Duomo giungono sino ai nostri giorni.

$* * *$

ABSTRACT. - The article offers an overview of the history of the cathedral of Milan in the context of the city. For a long period - at least until the end of the ancien régime - the Duomo housed the most important events of the city and was often the theatre of tensions between ecclesiastical and political authorities. The cathedral was the heart of the city and the center of pastoral activities and of religious life. Splendid ceremonies, often accompanied by music, took place in the Duomo, highlighting the importance of the bishops in the city. Even thouh in a different way compared to the past, some aspects of the rituality and centrality of the Duomo are still relevant today.

"Il Duomo si presenta alla storia come il cuore di Milano": ${ }^{1}$ in effetti è difficile pensare ad esso senza percepirlo come tale. Ciò è vero innanzitutto sul piano religioso; ma non dimentichiamo che, per secoli, in

(*) Università degli Studi di Milano, Accademia Ambrosiana di Studi Borromaici, Veneranda Fabbrica del Duomo-Milano.

1 E. Cattaneo, Il Duomo nella vita civile e religiosa di Milano, Milano $1985^{1}$. 
una società non secolarizzata, religioso e civile si fondevano inestricabilmente. Lo si constata persino nella scelta del luogo, non la sede naturale secondo le consuetudini del tempo, ma quello che era il luogo sacro già in età romana.

Non entro nel merito delle vicende della costruzione, né ho la presunzione di tracciare un quadro completo delle tappe che hanno segnato la vita del Duomo nel cuore della città, e della città attorno alla sua cattedrale. Piuttosto cercherò di restituire alcuni aspetti di ciò, soffermandomi con qualche dettaglio in più sulle vicende moderne.

Innanzitutto occorre ricordare che, a differenza di altri pur insigni monumenti nati dalla volontà di singoli o di piccoli gruppi, il Duomo si presenta come il "prodotto" della città, della comunità dei cittadini e dei fedeli. Gian Galeazzo nel 1401 prescrive che "essa chiesa in tutto e per tutto venga fatta, edificata e costruita secondo la volontà e le disposizioni dei suoi cittadini e uomini di Milano e dei deputati di detta Fabbrica". ${ }^{2}$ La costruzione fu arricchita dai grandi della città, a cominciare dagli arcivescovi; i ricchi nei loro testamenti disposero lasciti per la fabbrica e fondazioni di cappellanie; ${ }^{3}$ ma anche la gente comune apportò un significativo contributo ${ }^{4}$. Le oblazioni, provenienti da persone di ogni ceto, furono opportunamente sollecitate e incentivate nei momenti di maggiore difficoltà per la Fabbrica. Giovani e fanciulle, dalla fine del Trecento, usavano cantare per la città al fine di raccogliere offerte.

Un popolo che contribuisce alla costruzione della cattedrale la avverte come sua. Il popolo dei fedeli, pur con molte incongruenze e contraddizioni, possedeva - e così sarebbe stato almeno sino alla fine dell'epoca moderna - un forte senso della propria fede. Persino il peccato non era avvertito in contraddizione con ciò. Alcuni fedeli della diocesi milanese potevano, dunque, inoltrare una supplica a Roma firmandosi: "Peccatori, ma cristiani". Analogamente, i contributi per la Fabbrica vennero talora da persone di discutibile moralità, ma legate alla propria

2 Annali della Fabbrica del Duomo di Milano dall'origine fino al presente, 9 vol., Milano 1877-1885, vol. I, p. 141.

3 In età successiva, nei momenti di difficoltà e di conseguente diminuzione delle offerte, si sollecitarono i notai affinché "in occasione dei testamenti ricordino ai testanti, se vogliono fare qualche legato alla Fabbrica" (10 giugno 1649, Annali della Fabbrica, vol. V, p. 227).

4 Presso l'Archivio della Veneranda Fabbrica del Duomo di Milano è conservata preziosa documentazione in proposito. 
città e alla propria chiesa. Non a caso a Milano la parola Duomo prevale su cattedrale; il Duomo è la domus, la casa di tutti e di ciascuno, "la cà de Milan",; ma è anche "casa di Dio e porta del cielo". ${ }^{6}$

Il rapporto con le autorità civili nel tempo è stato costante, anche se non sempre facile. In un mondo nel quale, come si sa, gli eventuali conflitti tra autorità ecclesiastica e civile nulla toglievano a una radicale compenetrazione, gli episodi che si potrebbero menzionare sono numerosissimi. La cattedrale è il luogo ove avveniva l'ingresso solenne dei duchi, ove se ne celebravano i matrimoni, i battesimi, le esequie. Vi si recavano le autorità politiche che si avvicendavano. In particolare lo facevano, secondo quanto previsto dal protocollo, i governatori all'atto dell'insediamento. I sovrani in visita a Milano ottemperavano alla consuetudine cerimoniale di recarsi al Duomo. ${ }^{7}$ Così nel 1534 l'imperatore Carlo V si recò due volte in cattedrale, per pregare e per assistere alla messa. Per il medesimo imperatore in occasione della morte si ebbero, come si vedrà, straordinarie cerimonie funebri.

L'importanza attribuita alla cattedrale e la volontà politica di farne la sede specifica e particolare delle manifestazioni della sovranità peraltro non cessarono con la fine dell'antico regime. Seppur in un mondo assai diverso e con un differente significato, vi sono elementi di continuità. Su fondali del tutto mutati, lo spettacolo continua. E dunque Bonaparte al suo ingresso volle una cerimonia in Duomo e il canto di un solenne Te Deum, come si usava nel passato. Il mutamento è segnalato comunque anche dal fatto che l'arcivescovo Filippo Visconti non intendeva presenziarvi. Qui Napoleone fu incoronato con grande solennità il 23 maggio del 1805; il contesto era caratterizzato peraltro da accenti profani, tra applausi e accompagnamento di marce militari.

Un altro rifiuto sarebbe venuto dal card. Ferrari nel 1895. In contrasto con la Fabbrica (ligia al Governo) e in assoluta fedeltà a Leone XIII, l'arcivescovo non avrebbe voluto che, per la festa del 20 settem-

5 L'espressione in: E. De Marchi. El noster Domm (Milanin Milanon. Prose cadenzate milanesi, Milano 1926, pp. 57-63). In apertura e chiusura del testo, quasi identiche sono le parole: "In nomine patris, filii et spiritus sancti; l'è el noster Domm, l'è la gesa di vècc, l'è la cà de Milan, l'è tutt de màrmor, l'è grand, l'è bell, l'è lù, domà lù in tutt el mond, inscì bell, inscì grand".

6 Come ricorda G.B. Corno, Il Sacro Chiodo tesoro del Duomo di Milano, Milano, nella Stampa Archiepiscopale, 1647, p. 170.

7 A. Giulini, I genitori di Maria Teresa a Milano nel 1711 e 1713. Da diari inediti dell'epoca, Archivio Storico Lombardo, s. VI, 60 (1933), pp. 134-149. 
bre di recente istituzione, sulla guglia maggiore fosse issata la bandiera. I tempi erano radicalmente mutati, e anche il rapporto tra la cattedrale e la città non si presentava più nelle forme dell'età precedente.

I grandi eventi della vita politica e civile vi avevano a lungo trovato una eco consistente. Nel 1717, in occasione della vittoria di Eugenio di Savoia contro i turchi, l'imperatore aveva inviato alla città di Milano alcuni trofei di guerra, portati in cattedrale all'arcivescovo e appesi nello Scurolo. ${ }^{8}$ Nelle principali festività liturgiche le autorità civili assistevano alle sacre cerimonie. Ciò fornì a più riprese occasioni di conflitto. Si ebbero dissidi sulla collocazione delle autorità: ad esempio nella seconda metà del Cinquecento il conflitto si concentrò intorno alla disposizione della sedia e baldacchino del governatore, che costui voleva posta entro i cancelli del presbiterio. ${ }^{9}$ Nel dicembre 1756 il maestro delle sacre cerimonie, Nicola Lonari, attesta in una sua relazione i problemi insorti in occasione delle celebrazioni per la nascita dell'arciduca Massimiliano. ${ }^{10}$ La rappresentanza dell'autorità politica intendeva che il proprio baldacchino venisse posto più vicino all'altare rispetto a quello dell'arcivescovo e rialzato da una predella: una manovra che ostacolava l'accesso dei canonici agli stalli, ma che poneva soprattutto la questione dell'importanza dei convenuti. Alle obiezioni del cerimoniere su questa e altre problematiche la risposta fu la seguente: "Qui comanda Sua Altezza". Ciò nulla tolse allo splendore della "gran messa cantata a più cori da musici", ma ben documenta le difficoltà che Pozzobonelli sempre più incontrava nei suoi rapporti con il potere civile. La cattedrale è in certo senso luogo privilegiato del confronto e del conflitto. Così alla fine del $\mathrm{XV}$ secolo ebbe luogo, in occasione del ritorno dei francesi, una richiesta di perdono della città per le scelte politiche loro ostili. La richiesta si esplicò in una processione al Duomo di migliaia di bimbi che indossavano abiti bianchi, simbolo dell'innocenza. Essi implorarono pietà per i loro genitori e per la città intera, dinanzi al card. d'Amboise e ad altri illustri personaggi. ${ }^{11}$

8 A. Annoni, Gli inizi della dominazione austriaca, in Storia di Milano, vol. 12, Milano 1959, pp. 3-266 (p. 130s).

9 E. Cattaneo, Il Duomo, p. 115.

10 "Relazione del cerimoniere don Niccola Lonati sul cerimoniale della funzione celebratasi nel Duomo di Milano lì 26 dicembre 1756 per il parto dell'imperatrice Maria Teresa", ms., Biblioteca Ambrosiana, O 28 inf.

11 G.P. Bognetti, La città sotto i francesi. Aspetti politici, istituzionali e sociali, in Storia di Milano, vol. 8, Milano 1957, pp. 3-80 (p. 46). 
Gli apparecchi per le feste erano magnifici e costosi. Ciò vale in primo luogo per quelle celebrazioni che vedevano il coinvolgimento delle autorità pubbliche, di cui già dal XV secolo si hanno dettagliate descrizioni. Nel 1492, in occasione del battesimo del figlio di Gian Galeazzo Sforza, la cattedrale, adornata da drappi recanti la raffigurazione di ben 350 angeli, tra cherubini e serafini, si presentava alla vista degli astanti come una sorta di luminosa volta celeste. Per il matrimonio di Bianca Sforza con Massimiliano d'Austria nel 1493 la messa fu celebrata dall'arcivescovo "cum grandissime ceremonie et solemnitate de soni de trombe et pifferi, et organi et canti de la capella". ${ }^{12}$ Il cronista Burigozzo, descrivendo ornamenti e apparati effimeri con i quali era decorata la cattedrale in occasione dell'ingresso del duca nel 1534, afferma: "certo all'entrare de quella ecclesia pariva entrare in paradixo". Nella cerimonia funebre in suffragio di Carlo V venne affidata a Vincenzo Seregni la costruzione di un catafalco, adorno di complesse decorazioni, dipinte o in rilievo, e illuminato da 2250 ceri. Fino al 1780 per Maria Teresa, e poi ancora per Francesco I nel 1835, le ufficiature funebri dei sovrani furono celebrate con notevole solennità e grande afflusso popolare. Ciò avveniva spesso a considerevole distanza di tempo dal luttuoso accadimento, per permettere un'adeguata preparazione dell'evento, destinato a piangere il defunto, a esaltarne le virtù - rappresentate attraverso emblemi -, a celebrarne l'importanza, ma anche a coinvolgere la cittadinanza e dare gloria agli stessi organizzatori. Una cronaca del $1647^{13}$ mette in risalto il contrasto tra la città silente e la cattedrale che risuona di voci meste, di musiche e canti malinconici, spesso appositamente composti per l'occasione.

I funerali degli arcivescovi erano celebrati con grande solennità. Spicca fra tutti il funerale di Carlo, nel novembre del 1584; come è stato osservato, ${ }^{14}$ la predica di Francesco Panigarola e i canti appositamente composti da Giulio Cesare Gabussi si fondono perfettamente nei toni e nei contenuti, in sapiente orchestrazione, all'interno di una cerimonia affollatissima e coinvolgente. Si può notare che altre cerimonie funebri pure convogliarono al Duomo grandi folle; per tempi recenti si possono

12 A. Luzio, R. Renier, Delle relazioni di Isabella d'Este Gonzaga con Ludovico e Beatrice Sforza, Archivio Storico Lombardo, s. II, vol. VII, 17 (1890), pp. 74-119, 346399, 619-674, ivi pp. 384-388.

13 Breve racconto del sontuoso funerale [...], cit. in R.L. Kendrick. The sounds of Milan, 1585-1650, Oxford 2002, p. 478 n. 174.

14 Ivi, p. 153. 
ricordare i funerali di personalità laiche, come Arturo Toscanini e Gianni Versace, o nell'ambito ecclesiastico quelli di don Gnocchi, celebrati da Montini, e di don Giussani, cui partecipò anche l'allora cardinal Ratzinger.

Tradizionalmente sfarzosi erano gli apparati in occasione degli ingressi degli arcivescovi. Si erigevano archi trionfali, venivano collocate statue e iscrizioni, la facciata e l'abside erano adeguatamente illuminate. Il corteo di accompagnamento si snodava da Sant'Eustorgio al Duomo. Fogli volanti e cronache del Sei-Settecento ne descrivono le modalità. Aprivano abitualmente il corteo alcuni bambini, sovente vestiti da angeli. Tra le confraternite presenti, un ruolo sempre maggiore fu affidato alle scuole della dottrina cristiana, i cui membri, oltre a porgere il loro omaggio al novello arcivescovo, inscenavano veri e propri quadri allegorici a sfondo catechistico. Accanto allo stemma della Chiesa milanese figurava anche lo stemma della città: attraverso gli emblemi, il cui uso era tutt'altro che vuoto e formale, si prospettava il convergere dell'una e dell'altra, la simbiosi tra religione e civitas, talora presentate in tutte le loro articolazioni.

La cattedrale è il cuore liturgico e pastorale della vita religiosa. La liturgia vi era svolta con grande dignità e cura. Basti pensare ai cerimoniali per la messa pontificale cantata, che ancora nell'età contemporanea mostrano una ritualità articolata, complessa, stabilita sin nei più minuti aspetti. ${ }^{15}$ Il rito ambrosiano, peculiare e fortemente segnato dal rapporto con l'Oriente, caratterizza la tradizione locale e fu gelosamente conservato e difeso da Carlo Borromeo, in un'epoca nella quale si tendeva a sostituire i riti particolari con il romano. L'arcivescovo provvide anche a una riforma delle istituzioni della cattedrale e a una ristrutturazione della zona dell'altare, al fine di favorire lo svolgimento delle celebrazioni e far risaltare la centralità dell'eucaristia. L'azione dei vescovi post-tridentini necessitava di un supporto forte nell'edificio, spesso rinnovato al fine di adattarlo alle nuove esigenze. I dispositivi spaziali e gli oggetti necessari per le celebrazioni costituiscono il fondamento, materiale e spirituale allo stesso tempo, per lo svolgimento del

15 G.B. Germani, Messa pontificale arcivescovile cantata nella Metropolitana di Milano, Biblioteca Ambrosiana, ms., V 89 sup, ff. 31-38; su altre relazioni dello stesso autore: C. Castiglioni. Funzioni celebrate dall'arcivescovo di Milano secondo il diario del cerimoniere G.B. Germani, in Memorie storiche della diocesi di Milano, 11 (1964), pp. 433-443. 
culto. Anche l'arredo liturgico fu dunque arricchito in quantità e qualità, con l'acquisizione di oggetti talora di grande bellezza e valore, da custodirsi attentamente. Di qui il ruolo della sacrestia, su cui Carlo Borromeo insisteva, come una sorta di scrigno contenente cospicui tesori, che annuncia gli splendori delle molteplici e variegate funzioni e ne garantisce tanto la conformità alle norme quanto la magnificenza. ${ }^{16} \mathrm{Le}$ Instructiones fabricae, con la loro diffusione, fecero sì che tale orientamento divenisse patrimonio comune nell'Italia del Seicento. Tutto ciò mirava sia a valorizzare la figura del vescovo ed esaltarne la dignità, sia a permettere la degna celebrazione della liturgia e contribuire al sacrale decoro dei culti. D'altronde le cerimonie non sono fine a se stesse. Come scrive Juan de Maldonado nell'opera De Caeremoniis tractatus, ${ }^{17}$ esse svolgono un'essenziale funzione per la religione, analoga a quella del sale per i cibi, che li conserva e li rende più appetibili. Analogamente, la religione si mantiene e attira i cuori degli uomini attraverso l'apparato delle sue celebrazioni. $\grave{E}$ stato osservato che questo autore presenta la cerimonia come un discorso atto a convincere, come una "retorica in azione", che cattura i sensi, unendo la parola del rito e della predicazione, lo spettacolo visivo, i suoni vocali e strumentali, in primis dell'organo, le materie e i colori. A ciò si possono aggiungere gli odori, come il forte profumo dell'incenso che emana dai turiboli. La cattedrale diviene infrastruttura architettonica necessaria per lo svolgimento normale della vita religiosa, e al tempo stesso capolavoro di retorica visiva, atta a proiettare i fedeli verso la comprensione della religione e delle bellezze celesti. ${ }^{18}$ Quasi quattro secoli più tardi, Montini afferma: "L'impiego dei sensi è una conseguenza della nostra immissione nell'economia dell'Incarnazione, nella quale economia il mondo materiale diviene epifania, diviene linguaggio, mezzo cioè indispensabile per essere introdotti nel mondo invisibile e soprannaturale". ${ }^{19}$

Alla tutela del rito e alla cura per la vita liturgica si accompagnava la sottolineatura della centralità della cattedrale, riaffermata attraverso il divieto di celebrare nelle parrocchie durante lo svolgimento di cerimonie in Duomo. L'esaltazione della cattedrale, il suo perfetto ade-

16 G. Labrot, Sisyphes chrétiens. La longue patience des évêques bâtisseurs du Royaume de Naples (1590-1760), Seyssel 1999, p. 215.

17 Cit. Ivi, p. 207.

18 Ivi, passim.

19 Rivista diocesana milanese, 47 (1958), pp. 113-135. 
guarsi alla propria funzione, la sua bellezza, sono puntualmente delineati dagli arcivescovi nelle visite ad limina apostolorum.

Le cerimonie liturgiche attiravano non solo la popolazione, ma anche i forestieri e i viaggiatori (basti pensare che persino i quattro giovanissimi ambasciatori giapponesi di passaggio da Milano nel 1585 furono condotti a varie cerimonie religiose straordinarie).$^{20} \mathrm{~A}$ stimolare l'interesse contribuiva la musica. Come già era avvenuto alla metà del Quattrocento, potevano essere chiamati anche cantori stranieri; la sensibilità a varie esperienze italiane ed europee sarebbe stata a lungo una costante dell'attività musicale del Duomo. Dagli inizi dell'epoca moderna si hanno segnali di un costante adeguamento ai nuovi modi e al nuovo gusto, anche per attirare i fedeli. Tra i nomi di maggior rilevanza spicca quello di Franchino Gaffurio, tra la fine del Quattrocento e gli inizi del Cinquecento.

Il maestro di cappella ha una rilevante funzione, anche come compositore. In età moderna la cappella del Duomo continuò, seppur tra alti e bassi, la consolidata tradizione di dotarsi di maestri rinomati. Tra di essi spiccano alcuni nomi di rilievo, come quello di Vincenzo Ruffo nell'età di Carlo Borromeo. ${ }^{21}$ Tanto Carlo quanto Federico, anche se con accenti diversi, furono molto attenti alla musica come potente strumento di comunicazione e di rinnovamento religioso. La funzione della musica è dunque analoga a quella dell'architettura e delle arti visive, a noi più nota, poiché ancor oggi si può avere davanti agli occhi la magnificenza e il messaggio dell'apparato storico-artistico della cattedrale: basti pensare ai Quadroni di San Carlo che, esposti ogni anno, richiamano alla mente il significato e l'importanza di questa figura chiave, gli esiti della cui azione sono avvertibili per secoli e modellano la fisionomia della Chiesa locale.

Tornando alla musica. Carlo nel 1565 aveva ordinato a Ruffo, attraverso il suo vicario, di comporre una messa e di fargliene avere il testo. La richiesta prevedeva esplicitamente che si trattasse di un testo comprensibile e il più chiaro possibile, secondo l'idea di una 'musica intelligibile': proprio Ruffo è considerato un autore che mira alla piena comprensibilità, accanto alla gravitas. La volontà di san Carlo di realizzare una riforma liturgica e di ridare decoro alle sacre celebrazioni coinvolgeva anche l'aspetto della musica, tanto che la sua opera è considerata importante per

20 R.L. Kendrick, The sounds of Milan, p. 158 e note.

21 L.H. Lockwood, Vincenzo Ruffo and Musical Reform after the Council of Trient, The Musical Quarterly 43 (1957) n. 3, pp. 342-371; Cfr. sub voce, in Grove Music Online, ed. L. Macy, http://www.grovemusic.com. 
lo sviluppo della musica sacra della Controriforma. Anche i suoi carteggi attestano il puntuale interessamento a varie questioni (musica, figure istituzionali ecc.), sempre nel contesto del valore della liturgia. Le musiche, tanto vocali quanto organistiche, composte per il Duomo e ivi eseguite dal Quattrocento sino ai giorni nostri sono conservate in un'apposita sezione presso l'Archivio della Fabbrica, la cui importanza è illustrata anche dall'attenzione a ciò dedicata da illustri studiosi stranieri.

Nelle chiese di Milano fu attivo sin dal 1622 Michelangelo Grancini, ${ }^{22}$ poi maestro di cappella della cattedrale, rinomato per le sue composizioni polifoniche, con uno stile nuovo e molto espressivo ispirato a Monteverdi. Federico Borromeo fu particolarmente sensibile al significato della musica nei suoi aspetti estetici e pedagogici, sulla scia dell'orientamento di Filippo Neri.

Per limitarci ai nomi di spicco, ricordo che Johann Christian Bach durante il suo soggiorno milanese fu, dal 1760 al 1762, secondo organista della cattedrale. La fioritura della cappella si manifestò in tutto l'arco del Settecento, con maestri come Carlo Baliani e Giovanni Antonio Fioroni. ${ }^{23}$ Quest'ultimo, apprezzato anche da illustri personaggi stranieri, nella sua produzione mostra di saper fondere l'antico e il nuovo e attirare il consenso e il coinvolgimento del pubblico. È evidente che la dimensione liturgica era supportata e potenziata dalla musica, che contribuiva anche a riflettere e accrescere le devozioni, in assoluta circolarità.

La musica, in più semplici forme, accompagnava anche le numerosissime processioni dirette alla cattedrale. Così avveniva nelle processioni delle sei porte cittadine, cui partecipavano trombettieri e suonatori di oboe. Ogni porta giungeva alla cattedrale con i suoi stendardi, né mancava il gonfalone della città raffigurante il santo vescovo Ambrogio. Le processioni dirette alla cattedrale erano numerosissime. La ricorrenza della Natività di Maria vedeva la processione delle confraternite e corporazioni, che portavano le oblazioni, accompagnate da gonfaloni e trombettieri, nonché dai "trionfi", carri con raffigurazioni allegoriche. ${ }^{24}$ Altre si affermarono per ragioni diverse e divennero, come

22 J. Roche, Grancini, Michel'Angelo, Ibidem; Elenco dei maestri di musica le cui composizioni si conservano nell'Archivio musicale della Cappella del Duomo di Milano, in Annali della Fabbrica, Appendici II, pp. 139-206: Grancini alle pp. 169-180.

23 Si vedano Ibidem le varie voci biografiche e la voce Milan. Elenco dei maestri di musica: Baliani alle pp. 143-149, Fioroni alle pp. 160-167.

24 P. Ghinzoni, Trionfi e rappresentazioni in Milano (secolo XIV e XV), Archivio Storico Lombardo s. II, vol. IV, 14 (1887), pp. 820-831 (p. 827). 
nel caso dell'ingresso degli arcivescovi, vere e proprie occasioni di teatralità educativa. Noto è lo svolgimento di una processione del 1457 , che pose a tema la resurrezione e sviluppò l'arduo soggetto teologico attraverso felici allegorie. Esse risultavano comprensibili per tutti, poiché colpivano direttamente la mente e la fantasia: nella fattispecie, si pensi a Cristo che scoperchia il sepolcro, libera le anime del Limbo, abbatte il funesto nemico, Satana. I soggetti raffigurati nei quadri allegorici viventi erano a prevalente carattere sacro, ma non mancavano i temi profani, soprattutto a sfondo politico. Alcune processioni, come quella del 16 agosto 1452 , furono promosse dall'arcivescovo al fine di raccogliere fondi per la Fabbrica. ${ }^{25}$

Conformemente alla mentalità dell'epoca, la cattedrale - come anche le chiese e le vie stesse della città - divenne una sorta di "teatro", la cui funzione sacrale e profondamente educativa è del tutto evidente. Il rito stesso è, secondo le parole di Schuster, un "dramma sacro sui generis".

Il Duomo è dunque lo scenario all'interno del quale si costruisce un teatro sacro, finalizzato a coinvolgere emotivamente e, per questo tramite, a trasmettere i valori essenziali della fede, veicolati dalle immagini, dalle iscrizioni, dalle rappresentazioni, dalle celebrazioni sacre. Tutto ciò era destinato a un pubblico composito; sia le persone colte sia gli ignoranti potevano attingervi a seconda delle loro capacità e trarne spunti per un approfondimento della fede e della pratica religiosa. Il "diletto" si presenta dunque come via per l'apprendimento, secondo il noto binomio docere et delectare.

Un impulso notevole in questa direzione è riscontrabile in quella che gli storici oggi chiamano "età del rinnovamento cattolico", tra Cinque e Settecento. Nella sua incisiva ed energica azione Carlo Borromeo aveva puntato soprattutto sul primo aspetto, il docere, intendendo il secondo in funzione essenzialmente strumentale. Con lui la cattedrale diviene il "più severo palcoscenico" dell'azione di riforma; "il Duomo diventa innanzitutto la cattedra della verità cattolica applicata ad ogni settore anche minuto della vita pubblica e privata, dell'arte e del commercio". ${ }^{27}$ Si recupera in tal modo l'etimologia del nome "cattedrale", dalla cattedra del vescovo, luogo ove il pastore esercita le proprie funzioni,

25 G.B. Sannazzaro, Feste, in Il Duomo di Milano. Dizionario storico artistico e religioso, a cura di G. Benati e A.M. Roda, Milano 2001², pp. 264-268.

26 Rivista diocesana milanese 22, 1931, p. 534; 25, 1934, p. 539.

27 E. Cattaneo, Il Duomo, pp. 75 e 87. 
tra le quali rientra in primo luogo la proclamazione della verità cattolica. È, in ultima analisi, la proclamazione di un'identità riscoperta e approfondita, un'identità che dalla chiesa-edificio invade la vita. Il Duomo di ciò si fa simbolo e luogo.

Analogo concetto avrebbe espresso a distanza di secoli Giovanni Battista Montini: "Il Duomo è un posto di comando, un centro di scuola. Cattedra dice autorità e verità. La cattedrale è sede donde qualcuno governa ed insegna. Così il nostro Duomo: è il cuore vivo della Chiesa milanese. Non è soltanto un monumento inerte: è un organo pulsante", ${ }^{28}$ un'affermazione che a suo avviso, valida per Milano, si estende ben oltre i limiti della città.

In Duomo si svolgevano i sinodi (detti allora concili) diocesani e provinciali, i cui atti nell'età di Carlo Borromeo non solo segnarono le tappe della vita della Chiesa milanese, ma anche attirarono l'attenzione di molti vescovi; furono richiesti e imitati un po' ovunque, nell'Europa cattolica e persino nelle Americhe. Il numero di copie a stampa degli Acta Ecclesiae Mediolanensis fu enorme, con una diffusione del tutto eccezionale. Nei decreti si rifletteva la volontà di dare attuazione alla normativa del Tridentino, rispondendo alle necessità locali e combattendo al tempo stesso eventuali consuetudini contrarie. Il primo sinodo provinciale si tenne in cattedrale tra gli inizi di ottobre e gli inizi di novembre dell'anno 1565, con l'afflusso dei vescovi suffraganei, ben quattordici. Nell'arco di pochi anni si svolsero sei concili provinciali e undici diocesani. Essi continuarono poi a svolgersi in Duomo, con rarissime eccezioni.

Anche per quanto concerne la predicazione, il Duomo è un punto di riferimento: esso divenne il luogo principale - anche se non l'unico - della predicazione del vescovo; nell'Ottocento il card. Ferrari avrebbe ridato vigore a questa tradizione. In età moderna, altri illustri soggetti si alternavano sul pulpito, accuratamente controllati, spesso scelti tra cappuccini e gesuiti. Meglio sarebbe dire: sui pulpiti, poiché la cattedrale ne aveva due, ulteriore conferma, se necessario, dell'importanza attribuita alla predicazione. Molto richiesti erano i membri degli ordini mendicanti, che si avvicendarono con una certa frequenza sul pulpito e contribuirono alla diffusione di devozioni cristologiche e mariane. La predicazione dell'ordinario rivestiva peraltro l'importanza maggiore, sia

28 G.B. Montini, La Cattedrale, in Il nostro Duomo, Milano 1960, pp. n.n. tra pp. 24 e 26. 
per essere un fatto inconsueto, sia per la sua autorevolezza. Ciò vale sia per Carlo sia per Federico, la cui attività dal pulpito è fissata nella memoria anche dall'iconografia. L'arcivescovo attraverso la sua parola si presenta come l'incontrastata guida della vita religiosa, ma in ultima analisi - in un'epoca confessionale - anche di quella civile. La chiesa non cede dunque alla città, ma al contrario la dirige e la orienta. Non vi è un'accettazione più o meno supina della mentalità corrente e dello stato delle cose, ma si esprime una forza creativa, destinata a plasmare un nuovo modo di essere e dare origine a una socialità nuova. Così il suono delle campane del Duomo scandiva le ore della preghiera, giungendo nelle case e nelle botteghe.

Alla proclamazione "in positivo" si accompagnava la condanna dell'errore, come nel caso di coloro che erano caduti nell'eresia, posti nella chiesa in un luogo apposito e destinati a compiere per un lungo periodo una penitenza pubblica; essi dovevano recarsi alla cattedrale in abito penitenziale ed effettuavano la disciplina per tutta la durata della messa grande. ${ }^{29} \mathrm{La}$ cattedrale era in modo eminente anche il luogo della confessione. Per garantire l'amministrazione del sacramento anche agli stranieri, si derogò persino al divieto, imposto ai membri di ordini mendicanti, di confessare in Duomo: la deroga era concessa infatti nel caso di competenze in altre lingue, che il clero locale non possedeva. ${ }^{30}$

Come si sa, Carlo Borromeo non faceva concessione alcuna allo spirito profano nei luoghi di preghiera - che, come si è visto, si estendevano in certo senso all'intero spazio della città. Il desiderio di porre fine a pratiche religiose superficiali e abusive all'interno del Duomo è attestato ad esempio dal divieto del "cavallazzo", cioè di portare in cattedrale un cavallo di legno, adorno di vettovaglie. ${ }^{31}$ Anche i decreti dei sinodi diocesani indicano il desiderio di dare la prevalenza non alle profanità ma agli aspetti religiosi delle cerimonie e delle celebrazioni festive. $^{32}$ Nelle processioni erano ammessi gonfaloni e trombettieri, ma nulla doveva impedire la preghiera e la devozione. ${ }^{33}$

Sin dal 1566 la visita pastorale alla diocesi prese le mosse dalla vi-

29 E. Cattaneo, Il Duomo, p. 74.

30 Annali della Fabbrica, vol. III, p. 137 (18 marzo 1507).

31 E. Cattaneo, Il Duomo, p. 98.

32 Come attesta ad es. il Concilio Diocesano II, del 1568.

33 In conclusione degli Ordini del 22 maggio 1569: "Né si faccino in modo alcuno representationi, comedie, cavallacci, né altri simili spettacoli" (Annali della Fabbrica, vol. IV, p. 83s). 
sita alla cattedrale; è un dato che si ripresenta nel tempo, come si osserva ancora nel caso di Montini. Era condotta con grande cura e attenzione per gli aspetti anche più minuti. Infatti l'apparenza esteriore è importante. Chi giunge in cattedrale, già prima di entrarvi e poi all'interno, deve coglierne lo splendore epifanico e in tal modo percepire la sacralità del luogo e prepararsi a seguire e apprezzare i riti.

L'importanza attribuita da Carlo Borromeo al corretto svolgimento delle celebrazioni risulta anche dalla istituzionalizzazione della figura del maestro delle sacre cerimonie. I diari dei cerimonieri del Duomo, conservati presso l'Archivio capitolare, presentano un grande interesse per gli studiosi al fine di conoscere e comprendere la vivace e articolata ritualità che, a partire dalla cattedrale, si estendeva poi ad altre mete privilegiate, come S. Ambrogio o S. Maria dei Miracoli.

In Duomo Carlo Borromeo fondò alcune confraternite, come quella del SS.mo e quella del Rosario. La confraternita del Santissimo, aggregata all'arciconfraternita romana di S. Maria sopra Minerva, era impegnata nella promozione del culto eucaristico. Per accrescerne l'importanza, san Carlo la costituì come "Compagnia generale"; ad essa erano aggregati i membri delle varie confraternite del Santissimo presenti nelle parrocchie urbane, che potevano di conseguenza fruire delle numerose indulgenze concesse alla confraternita del Duomo.

I confratelli garantivano continuità di partecipazione e assicuravano la loro attiva presenza a svariate iniziative durante il corso dell'anno liturgico, come le Quarantore, la festività del Corpus Domini o il trasporto del viatico agli infermi. Alla confraternita si iscrissero figure istituzionali di spicco: il governatore spagnolo e vari membri della sua parentela. La potenza e solidità della confraternita fungeva da motore anche per la committenza artistica, soprattutto con una serie di tele raffiguranti i più rinomati miracoli eucaristici. Esse venivano esposte in occasioni particolari, come ad esempio nel 1895 in concomitanza con la celebrazione del $13^{\circ}$ Congresso Eucaristico Nazionale.

Il culto eucaristico ebbe grande sviluppo nell'epoca moderna, con tabernacoli e ostensori sempre più decorati. Si affermò la centralità del tabernacolo e la conservazione dell'eucaristia sull'altar maggiore, cuore vivente e visibile della cattedrale; al tempo stesso, si moltiplicarono gli altari per la celebrazione delle messe. La pratica dell'ostensione dell'eucaristia e le Quarantore - che si può definire un' autentica "missione devozionale urbana" - ebbero inizio negli anni venti-trenta del Cinquecento, ma furono poi istituzionalizzate e potenziate da Carlo Borromeo. Sottolineando volutamente la centralità del Duomo, il Borromeo 
dispose che alle quarantore celebrate in cattedrale partecipassero il clero regolare e secolare e le confraternite urbane, con il loro seguito di fedeli. Merita osservare che non si tratta di pratiche intimistiche. L'eucaristia infatti, carnale presenza di Dio tra gli uomini, consente il più alto punto di incontro tra l'uomo e Dio e al tempo stesso crea la comunità dei fedeli e li apre al mondo, nel quadro del "primato cattolico della parola incarnata e socializzata", per usare una felice espressione di Marc Fumaroli. ${ }^{34}$ Il fasto dei tabernacoli indica il prezioso contenuto, gli ostensori ne consentono l'adorazione attraverso la contemplazione visiva, documentandone anche l'irraggiamento esterno.

La processione del Corpus Domini, momento essenziale della vita civile e religiosa della città, ebbe inizio dal 1335 e si rivestì di sempre maggiore solennità. Si snodava verso la cattedrale su un percorso prestabilito, con la partecipazione dei corpi religiosi e delle autorità civiche, in strade addobbate a festa e con un pregevole accompagnamento musicale. Dopo l'interruzione durante il periodo della Cisalpina, la consuetudine riprese, ma evenienze politiche o scelte pastorali spesso indussero a restringerla all'ambito della cattedrale o a rivederne i percorsi. ${ }^{35}$ Il Borromeo aveva istituito anche qui l'usanza, come in tutte le chiese parrocchiali, di una processione eucaristica ogni terza domenica del mese, cui i membri della confraternita assicuravano il loro accompagnamento.

La confraternita del Rosario, fondata nel 1584, aveva la finalità di accrescere la devozione mariana attraverso questa forma di preghiera, in sintonia con la Sede romana. Il pontefice Pio V ne aveva infatti istituita la festa a seguito della battaglia di Lepanto. La fondazione della nuova confraternita in Duomo - anche se la devozione del rosario era tradizionale appannaggio dei domenicani - è uno degli argomenti af-

34 Così M. Fumaroli, citato da C. Leri, Dilettare e istruire. San Carlo nella poesia barocca lombarda, Studia borromaica. Saggi e documenti di storia religiosa e civile della prima età moderna, 13 (1999), pp. 235-266, ivi p. 235. La celebrazione del Corpus Domini: donne à voir non seulement l'hostie mais aussi toute la société réunie dans une commune adoration (O. Chaline, La reconquête catholique de l'Europe centrale XVIXVIII siècle, Paris 1998, p. 90).

35 Durante gli episcopati di Colombo e Martini vi furono ripensamenti notevoli, sino alla decisione di Martini di mutare per alcuni anni la destinazione, non più il Duomo, ma luoghi vari scelti di volta in volta. Cfr.: P. Vismara. La "pietas" ambrosiana in Storia di Milano, Roma 1996, vol. XVIII/2, pp. $622-632$ (p. 622). 
frontati nel carteggio tra Borromeo e Cesare Speciano nel marzo-aprile del 1584, a dimostrazione dell'importanza a ciò attribuita. Altra confraternita mariana di origine borromaica è quella dell'Assunzione, che riuniva "sessanta matrone" ed era dotata di numerose indulgenze.

In cattedrale esistevano anche altre confraternite, alcune delle quali fondate in epoca anteriore, come quella dell'Ave Maria (detta anche "del pilone"), di S. Maria della Neve, di S. Giovanni in Fonte, dei Santi Rocco e Vittore, e via dicendo. La devozione alla Madonna "del pilone" era così intensa che l'arcivescovo Carlo intervenne per istituzionalizzarla (e in ultima analisi controllarla), mediante un trasferimento all'altar maggiore. Gli interventi regolatori si concentrarono anche sulla Madonna "del coazzone", assai venerata, ma forse con qualche disordine, tanto che la statua, posta su un altare laterale, fu tolta per evitare abusi e superstizioni.

Le confraternite sovente provvedevano a far eseguire a loro spese della musica, nonché ad accendere ceri. Come ricerche recenti hanno efficacemente illustrato, l'aspetto della luce era fondamentale, per quanto concerne sia la luce naturale filtrata dalle vetrate, sia l'illuminazione vera e propria: la luce materiale e terrena, oltre a mettere in evidenza la ricchezza e la sontuosità dell'edificio, prefigura infatti quella celeste. Le disposizioni erano molto precise, come documentano i decreti che specificano nel dettaglio i ceri da accendersi quotidianamente ai vari altari.

Tra le confraternite, peculiarmente borromaica era quella della dottrina cristiana. ${ }^{36}$ Un corteo interessante e significativo accompagnò, nel 1748, un soldato ebreo convertito da S. Sepolcro alla cattedrale, ove egli avrebbe ricevuto i sacramenti per mano dell'arcivescovo. L'uso degli spazi del Duomo fu orchestrato in modo sapiente: il battesimo impartito nel battistero, la cresima all'altar maggiore, la prima comunione allo Scurolo di San Carlo. La cerimonia fu accompagnata da brevi rappresentazioni, con forte intento pedagogico e catechetico, affidate alle scuole della dottrina cristiana.

Le processioni, cui già ho fatto cenno, sono un elemento degno di nota. Alcune ebbero vita relativamente breve, come la processione dell'arca, scomparsa nel tardo medioevo, ma interessante per l'influsso esercitato sugli usi liturgici ambrosiani, alle origini della Chiesa milanese, da

36 Si vedano le raffigurazioni relative all'ambiente milanese nei quadri di Magnasco, in cui alcuni particolari richiamano proprio il Duomo: Alessandro Magnasco (1667-1749), Milano 1996, pp. 200-202. 
tradizioni ebraiche filtrate da missionari orientali. Altre si svolsero in ricorrenze festive eccezionali, come nel caso dei giubilei, oppure nel 1751 in occasione della sistemazione dello Scurolo e della traslazione del corpo di san Carlo. La tomba di Carlo era un centro di sentita devozione, ove tra l'altro si raccoglieva gran numero di offerte, ${ }^{37}$ vi si manifestava il senso di fedeltà alla Chiesa ambrosiana e alla sua storia.

Processioni mariane erano affidate alla confraternita del Rosario: dal Duomo fin sulla piazza si trasportava con grande solennità lo stendardo mariano, $o$, nelle feste mariane più importanti, una statua riccamente ornata. Nella festa liturgica della Purificazione di Maria si celebrava un tempo la processione della Madonna "Idea", inizialmente da S. Maria Beltrade al Duomo, e, dalla fine del Cinquecento, al suo interno.

La reliquia del sacro Chiodo era assai venerata, sin dal medioevo; il suo trasferimento dall'antica cattedrale alla nuova avvenne con grande solennità e massiccio afflusso di fedeli e la reliquia fu posta nella volta dell'abside. Nel 1576, in occasione della peste, Carlo Borromeo trasportò il sacro Chiodo in processione sino al santuario di S. Maria dei Miracoli, con l'accompagnamento solenne del clero regolare e secolare: tutti recavano la più insigne reliquia delle loro chiese. Al ritorno in Duomo, il sacro Chiodo fu esposto sull'altare per 40 ore consecutive, come si usava fare per l'eucaristia: all'adorazione si unì un ciclo ininterrotto di predicazione. Negli archivi si conservano elenchi di predicatori, che si alternavano in quell'arco di tempo. ${ }^{38}$ Non è da trascurare il significato di questo simbolo, che si connette direttamente al crocefisso, il cui valore ad un tempo teologico ed affettivo è ricordato ad esempio da Giusto Lipsio nel De cruce. ${ }^{39}$

Da tale avvenimento conseguì una maggiore devozione per la reliquia, con una processione esterna ${ }^{40}$ e varie celebrazioni in cattedrale fissate al 3 maggio, festa liturgica dell'Invenzione della croce. Il Corno descrive con puntuale attenzione il decoro: "La Veneranda Fabbrica del Duomo l'anno 1625 fece dipingere tutta la volta del choro d'azuro con

37 Ad es.: Archivio Storico Diocesano di Milano, Fondo Visite Pastorali, Metropolitana, vol. 31, q. 15; vol. 49, q. 3.

38 Ad es.: Ivi, vol. 29, q. 13, vol. 82, q. 9.

39 Cit. in O. Chaline. La bataille de la Montagne Blanche (8 novembre 1620). Un mystique chez les guerriers, Paris 2000, p. 306.

40 La meta successivamente divenne la chiesa di S. Sepolcro. Cfr. G.B. Corno. Il Sacro Chiodo, passim (ad es. pp. 74-76 per la processione del 1602 con l'arcivescovo Federico Borromeo). 
stelle di rame indorate, quali rendono una vista mirabile, e fece far un ornamento intorno al Sacro Chiodo di dodici angeli dello stesso metallo indorati, duoi de quali più vicini alla Reliquia sostengono una corona de spini, duoi altri si vedono, uno con il martello, l'altro con la tenaglia, e duoi altri, che sono i più grandi, tengono in ciascuna mano un toribolo: gli altri poi sono in atto di adoratione, et è ornamento tanto maestoso, che pare a spettatori un paradiso".$^{41}$

Tali celebrazioni, arricchite da indulgenze pontificie, ${ }^{42}$ furono molto frequentate e diedero luogo a composizioni musicali come ad esempio Il sacro santo e fortunato Chiodo di Gasparo Costa, noto autore anche di mottetti e madrigali spirituali. Il rito della "nivola", ancor oggi in vigore, accresceva l'interesse popolare. Un'altra ragione di afflusso era determinata dalla convinzione che l'esposizione della preziosa reliquia consentisse la guarigione dei malati, in particolare degli ossessi; coloro che erano ritenuti tali vi erano dunque accompagnati in buon numero. ${ }^{43}$ La notorietà della potenza taumaturgica della reliquia varcava $\mathrm{i}$ confini; i fedeli vi accorrevano ex omnibus finitimis status et provinciae civitatibus et oppidis, secondo le parole dell'arcivescovo Erba Odescalchi in una sua visita ad limina. L'afflusso anche dall'estero è attestato tra l'altro da una lettera di raccomandazione scritta da Domenico Passionei, nunzio in Svizzera e futuro cardinale, e indirizzata a Giuseppe Antonio Sassi. L'austero prelato scriveva da Altdorf il 23 aprile del 1728: "Il renditore di questa mia è un poveretto d'una parocchia qui vicina, il quale sentendosi da molto tempo travagliato da gravissimi mali, se ne viene espressamente a Milano per esser presente alla mostra, che si farà del Sacro Chiodo, con grandissima fiducia di riportarne sollievo; ma siccome vorrebbe la consolazione di poter vedere e venerar davvicino così eccelsa reliquia, perciò la raccomando particolarmente alla sua carità, affinché si prenda la pena di farlo collocare in qualche luogo com-

41 Ivi, pp. 124-125.

42 Ivi, passim.

43 "Scaccia i demonii e la peste"; per il Sacro Chiodo "sono cruciati e tormentati li demonii" (Ivi pp. 21 e 170). Per il controllo civile in merito al fenomeno degli ossessi durante le sacre cerimonie del 3 maggio, cfr. P. Vismara. Religione, superstizione, medicina in un episodio settecentesco, Studi e fonti di storia lombarda 6 (1986) 11, pp. 21-26. Nell'opera Della forza della fantasia umana, Muratori cita i fenomeni ricorrenti durante queste cerimonie quale esempio del meccanismo per il quale "la fantasia guasta di una donna se ne tira dietro cento altre" (cito dalla ed. delle Opere, per Michele Bellotti, Arezzo 1768, t. VII, p. 267). 
modo, ond'egli possa rimaner soddisfatto" ${ }^{44}$ Anche le autorità politiche a lungo garantirono la propria presenza: almeno sino a che la diffidenza e il disprezzo non ebbero il sopravvento nell'età giuseppina.

Una caratteristica peculiare delle processioni con il sacro Chiodo, all'interno o all'esterno del Duomo, è il fatto che, nell'una o nell'altra forma, esse furono effettuate quasi senza soluzione di continuità, anche se con il tempo le solennità divennero assai più modeste e la data fu mutata, con il trasferimento al 14 settembre. ${ }^{45}$

Soprattutto nel caso di pestilenze, ma più in generale nei momenti di difficoltà, la cattedrale si presentava davvero come un punto di riferimento essenziale ed ineliminabile per la città. Il crocefisso (ancor oggi detto di San Carlo o della peste) fu portato in processione durante l'epidemia del 1576; la continuità tra passato remoto e prossimo è ben documentata da almeno due processioni novecentesche, in occasione di altri gravi avvenimenti: con Schuster durante la seconda guerra mondiale, e con Montini, nel 1956, in occasione della sanguinosa repressione dell'insurrezione di Ungheria.

$\mathrm{Al}$ contrario, non vi è continuità nei rapporti con l'autorità politica, che nell'Ottocento si presentano come stabilmente - o quasi - conflittuali. Le interferenze politiche erano numerose. Tanto per fare un esempio, alla morte dell'arcivescovo Nazari di Calabiana, avvenuta nel 1893, non fu autorizzata la sepoltura in Duomo: ciò sarebbe stato possibile solo nel $1912 .{ }^{46} \mathrm{Nel} 1895 \mathrm{fu}$ vietata dalla prefettura una processione che avrebbe dovuto svolgersi da S. Ambrogio alla cattedrale, in occasione del congresso eucaristico nazionale. Il divieto era motivato con "ragioni di ordine pubblico". ${ }^{47}$ In ogni caso, frequentatissime furono le cerimonie inaugurali e conclusive, cui parteciparono due cardinali e più di sessanta vescovi.

Come nel 1895, anche nel 1983 fu celebrato a Milano il congresso eucaristico nazionale; alcune cerimonie liturgiche si svolsero in Duomo; altre, per ragioni logistiche e l'elevatissimo afflusso di fedeli dovuto alla presenza di Giovanni Paolo II, furono celebrate sulla piazza o addirit-

44 Bibl. Ambrosiana, ms., Z 210 sup., n. 238. Per l'afflusso di non milanesi, Cfr. G.B. Corno. Il Sacro Chiodo, pp. 97 e 187.

45 Un'affollata processione pubblica ebbe luogo durante l'episcopato del card. Martini, nel 1984.

46 E. Cattaneo, Il Duomo, p. 152.

47 F. Ruggeri, Congressi eucaristici, in Il Duomo di Milano. Dizionario, pp. 188-190. 
tura in aree periferiche. L'anno successivo il pontefice ritornò a Milano per celebrare il santo arcivescovo Carlo e pregare sulla sua tomba.

Afferma Montini parlando del Duomo: "Qui è il centro della pietà milanese, qui è il cuore della diocesi, qui è il faro della sua unità, qui la radice della sua ultima tradizione: qui vi è una sorgente di vita, tuttora sgorgante" ${ }^{48}$ In effetti egli intendeva il Duomo, come già si è detto, come il luogo della proclamazione della verità da parte dell'arcivescovo; come il luogo nel quale il culto liturgico doveva svolgersi in modo esemplare, nella consolidata fedeltà alla tradizione e a uno spirito autenticamente religioso ${ }^{49}$ infine, come "il segno visibile della centralità di Cristo nella storia, elemento unificante di una realtà che tende a scomporsi" ${ }^{50}$ La cattedrale esprime una forza centripeta, cui consegue parallelamente un moto verso l'esterno. Da qui, da questo centro e questo cuore, partì la "Missione di Milano" nel 1957; da essa centinaia di predicatori si mossero per portare alla civitas "il tesoro vitale delle nostre verità". ${ }^{51}$

Giovanni Paolo II nella sua visita del 1983 parlò del Duomo come di "simbolo celebrato della fede e della civiltà di Milano". ${ }^{52}$ Fulcro della vita della città, esso svolge ancor oggi un ruolo altamente simbolico, che potrebbe essere ulteriormente valorizzato pur in una società laica, poiché la Chiesa si presenta come "maestra di umanità". Si può forse dire che tale orientamento è espresso in modo simbolico già dalla costruzione. Le terrazze, inconsuete in una cattedrale gotica, aprono sulla città e sul territorio, sotto lo sguardo vigile della Madonnina.

Sin dall'inizio la cattedrale fu dedicata a Maria; secondo la tradizione, il voto dei milanesi alla Madonna risale all'epidemia pestilenziale del 1386. Dando seguito a un progetto avanzato secoli prima, nel 1774 sulla guglia maggiore fu posta la "Madonnina", tanto cara al cuore dei

48 G.B. Montini, La Cattedrale.

49 I. Biffi, L'arcivescovo Montini e la liturgia, in G.B. Montini arcivescovo, a cura di A. Majo, Milano 1983, pp. 95-135.

50 G. Rumi, Montini, Giovanni Battista. Storia, in Il Duomo di Milano. Dizionario, pp. 376-377.

51 Cit. da G. Rumi, Una cattedra tra Milano e Roma, in Storia di Milano, Roma 1996, vol. XVIII/2, pp. 591-606, ivi p. 601.

52 In: E. Cutolo, Il papa a Milano, Città del Vaticano 1983, p. 173. Il pontefice parlò anche del "magnifico Duomo" come di "vero inno di gloria dell'arte cristiana, segno vivo della fede eucaristica d'un popolo, testimone ed espressione di secoli di storia e di pietà, tempio insigne che in qualche modo - come ebbe a dire Paolo VI - 'definisce, qualifica la città, ne perenna le imprese più nobili' ” (Ivi, p. 152). 
milanesi. ${ }^{53}$ Anche se non vi sono precise attestazioni in materia, non si può a mio avviso escludere che si tratti di una riconquista simbolica dello spazio celeste, quando gli altri spazi erano sempre più controllati e reclamati dal potere civile.

Claudel la chiama "santa vergine dorata risplendente al sole"; altri, in modo meno dotto e in lingua milanese, la cantano con bonaria "milanesità". Ricordo ad esempio E pur mi disi di Valter Valdi, che così spiega il frenetico attivismo dei milanesi: è "la Madonina /che a la mattina / senza fass vedè, / la spara on colp in aria / e la dà el via. / E numm se gh'emm de fa? / Sentom sparaa... / e alé, 'taccom andà". Anche queste sono espressioni del legame tra la città e la Madonnina: "Madonna che parla in milanes", secondo Nino Rossi, "nòster sorris, s'giaffa de sô in mezz a "sto gris". ${ }^{54}$

Alla "aurea Madonnina" si rivolgeva con parole affettuose e vibranti il card. Schuster al termine della guerra. Emblematica per la comunità cittadina fu, nel maggio del 1945, la cerimonia con la quale egli scoprì la statua, che era stata opportunamente coperta e nascosta onde evitare che costituisse con la sua lucentezza un punto di riferimento negli attacchi aerei. Di protezione della città parla anche Montini: "La Madonnina aleggia su la città: col suo gesto la benedice, col suo volo la solleva in alto, col suo sguardo al cielo la protegge e la salva";5 è un "celeste emblema" che "farà sì che questa resti sempre una città cristiana ed una città di luce", ${ }^{56}$ proteggendo tanto la comunità cristiana quanto la civitas. Nonostante tutto, tra sorrisi e preghiere ad essa forse guardano ancora molti abitanti di questa città che muta volto ma conserva alcuni riferimenti essenziali, in un legame talora fragile, mai del tutto interrotto.

53 "Il rettore partecipa di aver fatto indorare e collocare in cima alla gran guglia la statua della Beata Vergine, e che quest'opera ha meritato l'universale applauso" ( $A n$ nali della Fabbrica, 30 dicembre 1774, vol. VI, pp. n.n.). Negli anni precedenti si erano svolti dibattiti ed erano stati presi provvedimenti (con l'intervento del Boscovich e del torinese padre Beccaria, noto studioso di elettricità) per difendere la guglia maggiore dal pericolo dei fulmini: Annali della Fabbrica, vol. VI, p. 192 (1770) e 194 (1773); G.B. Beccaria, "Maniera di preservare dal fulmine il Duomo di Milano", Milano 21 ottobre 1770, ms, Biblioteca Ambrosiana, L 42 inf n. 8. Nella sua relazione, accompagnata da figure illustrative, lo scolopio ricorda tra l'altro che già "avanti che si alzasse la grande piramide sopra la cupola del Duomo, a memoria nostra due volte è stata fulminata e danneggiata una molto più bassa piramide". Nella nuova situazione, la mancanza di conduttori per i fulmini potrebbe "trarre seco la rovina dell'ammirabile edificio".

$54 \mathrm{http} / /$ www.canzon.milan.it/madonina.htm

55 G.B. Montini, La Cattedrale.

56 Discorso tenuto in Duomo, 8 dicembre 1960, ora in: G.B. Montini. Sulla Madonna. Discorsi e scritti (1955-1963), Brescia-Roma 1988, p. 158. 